\title{
On the Resolution of Inhomogeneous Norm Form Equations in Two Dominating Variables
}

\author{
By István Gaál*
}

\begin{abstract}
Applying Baker's well-known method and the reduction procedure described by Baker and Davenport, we give a numerical algorithm for finding all solutions of inhomogeneous Thue equations of type

$$
N_{K / Q}(x+\alpha y+\lambda)=1
$$

in the variables $x, y \in Z$ and $\lambda \in Z_{K}$ with $\left.\lambda<(\max |x|,|y|)\right)^{1 / 2}$, where $K=Q(\alpha)$ is a totally real cubic field.
\end{abstract}

1. Introduction. In 1966 Baker developed a new method (cf., e.g., [2]) for solving Diophantine problems which led to effective upper bounds for the solutions of wide classes of Diophantine equations (for a survey, see, e.g., [23] and [20]). Here we restrict ourselves to norm form equations, and we mention only the results of Baker [1] (cf. also [2]) and Coates [4] on Thue equations and the theorems of Györy and Papp [13], Györy [9]-[12] and Kotov [15]-[17] on norm form equations in several variables.

In 1974 Sprindžuk [21] gave a so-called inhomogeneous generalization of Baker's famous result on Thue equations. Let $\alpha$ be an algebraic integer of degree $\geq 3$, $K=Q(\alpha), 0 \neq m \in Z$. Consider the equation

$$
N_{K / Q}(x+\alpha y+\lambda)=m,
$$

where the variables are $x, y \in Z$ and ${ }^{* *} \lambda \in Z_{K}$. To ensure the finiteness of the number of solutions of (1) it is necessary to restrict the values of $\lambda$. Sprindžuk assumed that ${ }^{* * *}|\lambda|<(\max (|x|,|y|))^{1-\xi}(0<\xi<1$ is a given constant $)$. Thus $\lambda$ may be called a nondominating variable, while $x$ and $y$ are dominating variables. Under the above conditions, Sprindžuk derived effective upper bounds for all solutions of Eq. (1). In the special case $\lambda=0$, his result implies Baker's theorem on Thue equations.

Later, combining the method of Györy and Papp [13] and Sprindžuk [21], the author [7], [8] obtained effective upper bounds also for the solutions of certain inhomogeneous norm form equations in several variables.

Received January 5, 1987; revised October 26, 1987.

1980 Mathematics Subject Classification (1985 Revision). Primary 11Y50; Secondary 11D57.

Key words and phrases. Computer solution of Diophantine equations, Thue equation, Davenport's lemma.

*Research (partially) supported by the Hungarian National Foundation for Scientific Research, Grant No. 273.

${ }^{* *} Z_{k}$ denotes the ring of integers of an algebraic number field $K$.

${ }^{* * *}$ As usual, $|\lambda|$ denotes the size of an algebraic number $\lambda$, that is, the maximum absolute value of its conjugates. 
The problem of solving the Thue equation

$$
N_{K / Q}(x+\alpha y)=m \quad \text { in } x, y \in Z
$$

can be stated in the following way: Determine all elements in the $Z$-module $\{1, \alpha\}$ with given norm $m$. On the other hand, the resolution of Eq. (1) means to find all elements of $Z_{K}$ which have norm $m$ and which are not too far from the elements of the $Z$-module $\{1, \alpha\}$. Moreover, inhomogeneous equations of type (1) have important applications in the theory of Diophantine approximations (cf. [7] and [8]). Finally, we observe that for any fixed $\lambda$, Eq. (1) is an inhomogeneous polynomial equation in the variables $x, y \in Z$. Previously there was no general method of handling such inhomogeneous equations, but Sprindžuk's result, as a special case, gives effective upper bounds for all solutions $x, y$ of these equations.

All the results mentioned above yield only a theoretical solution of the Diophantine problems, since the upper bounds derived for the solutions by using Baker's method are usually very high and in most cases, even with the fastest computers, it seems impossible to check all values of the variables below this bound. To overcome this difficulty, Baker and Davenport [3] described a new computational method, which, in numerical cases, usually allows one to reduce the large upper bounds and to find all solutions of certain Diophantine equations. Their method was applied for solving Thue equations and was further refined and extended by Ellison [5], Ellison et al. [6], Steiner [22] and Pethö and Schulenberg [19]. Steiner, Pethö and Schulenberg utilized the observation that, with the exception of few small values, all solutions of Thue equations $\left(1^{\prime}\right)$ correspond to partial quotients in the continued fraction expansion of the real conjugates of $\alpha$. This idea enabled Pethö and Schulenberg to solve also Thue equations of degree higher than three. Recently, Pethö [18] gave an efficient algorithm to determine all solutions of Thue equations up to a large bound.

In this paper we apply the method of Baker and Davenport to the resolution of inhomogeneous Thue equations of type (1). For simplicity we restrict ourselves to the case when $K$ is a totally real cubic field, $\xi=1 / 2$ and $m=1$. (We remark that our method can be modified to work for any algebraic number field $K$ with small number of fundamental units, for any $m$ and for any $\xi \geq 1 / 2$.) We illustrate our algorithm on a numerical example.

2. Effective Upper Bounds Using Baker's Method. We consider the equation

$$
N_{K / Q}(x+\alpha y+\lambda)=1 \quad \text { in } x, y \in Z \text { and } \lambda \in Z_{K},
$$

where $K=Q(\alpha)$ is a totally real cubic field. Putting $X=\max (|x|,|y|)$, our condition on $\lambda$ can be written in the form $\lambda<<X^{1 / 2}$. In our numerical example, $\alpha$ is defined by the equation

$$
x^{3}-3 x^{2}-4 x-1=0
$$

and the approximate values of its roots are

$$
\alpha^{(1)}=-0.69202147, \quad \alpha^{(2)}=-0.3568958, \quad \alpha^{(3)}=4.0489173 .
$$


We shall denote by $\gamma^{(i)}$ the conjugates of any $\gamma \in K$ corresponding to $\alpha^{(i)}$. Let $\beta^{(i)}=x+\alpha^{(i)} y+\lambda^{(i)}(i=1,2,3)$. Using this notation, Eq. (2) has the form

$$
N_{K / Q}(\beta)=\beta^{(1)} \beta^{(2)} \beta^{(3)}=1 .
$$

In our example, a pair of fundamental units of $K$ with norm +1 is $\eta_{1}=\alpha$, $\eta_{2}=-\alpha^{2}+3 \alpha+2$. In view of $(3), \beta=\eta_{1}^{b_{1}} \eta_{2}^{b_{2}}$ with some $b_{1}, b_{2} \in Z$.

In this section our main purpose is to give an upper bound for $H=\max \left(\left|b_{1}\right|,\left|b_{2}\right|\right)$. We shall apply the method of Sprindžuk [21] and some ideas of Steiner [22] as well. For $1 \leq k, l \leq 3, k \neq l$ we have

$$
\begin{aligned}
& \log \left|\beta^{(k)}\right|=b_{1} \log \left|\eta_{1}^{(k)}\right|+b_{2} \log \left|\eta_{2}^{(k)}\right|, \\
& \log \left|\beta^{(l)}\right|=b_{1} \log \left|\eta_{1}^{(l)}\right|+b_{2} \log \left|\eta_{2}^{(l)}\right|
\end{aligned}
$$

whence

$$
H \leq N \max \left(\log \left|\beta^{(k)}\right|, \log \left|\beta^{(l)}\right|\right),
$$

where $N$ is the row-norm of the inverse matrix of

$$
\left(\begin{array}{cc}
\log \left|\eta_{1}^{(k)}\right| & \log \left|\eta_{2}^{(k)}\right| \\
\log \left|\eta_{1}^{(l)}\right| & \log \left|\eta_{2}^{(l)}\right|
\end{array}\right)
$$

that is, the maximum sum of the absolute values of the elements in its rows. Choosing appropriate values of $k, l$ which minimize $N$, we get in our example $N=2.6614048$. Further, (4) implies

$$
H \leq N \log \left[\left(2+\max _{i=k, l}\left|\alpha^{(i)}\right|\right) X\right]
$$

that is,

$$
\log X \geq c_{1} H-c_{2}
$$

where $c_{1}=1 / N$ and $c_{2}=\log \left(2+\max _{i=k, l}\left|\alpha^{(i)}\right|\right)$. In our example, we have $c_{1}=0.3757414, c_{2}=0.9902923$. Applying the method described in Section 5, we searched over the range $H \leq H_{0}$ (in our example, $H_{0}=35$ ) to find solutions. Thus we must deal here only with those pairs $b_{1}, b_{2}$ for which $H>H_{0}$, whence, in view of the last estimate, we may assume that

$$
X \geq \exp \left(c_{1} H_{0}-c_{2}\right)=X_{0},
$$

where in our example $X_{0}>191119$. Let

$$
\left|\beta^{(i)}\right|=\max _{k=1,2,3}\left|\beta^{(k)}\right| \text { and }\left|\beta^{(j)}\right|=\min _{k=1,2,3}\left|\beta^{(k)}\right| .
$$

Obviously $\left|\beta^{(j)}\right| \leq 1$ (cf. (3)), and thus

$$
\left|\beta^{(i)}\right| \geq\left|\beta^{(i)}-\beta^{(j)}\right|-\left|\beta^{(j)}\right| \geq\left|\alpha^{(i)}-\alpha^{(j)}\right||y|-2 X^{1 / 2}-1
$$

Further, since

$$
\alpha^{(j)} \beta^{(i)}-\alpha^{(i)} \beta^{(j)}=\left(\alpha^{(j)}-\alpha^{(i)}\right) x+\alpha^{(j)} \lambda^{(i)}-\alpha^{(i)} \lambda^{(j)},
$$

we get

$$
\left|\beta^{(i)}\right| \geq \frac{\left|\alpha^{(j)}-\alpha^{(i)}\right|}{\left|\alpha^{(j)}\right|}|x|-\frac{\left|\alpha^{(i)}\right|+\left|\alpha^{(j)}\right|}{\left|\alpha^{(j)}\right|} X^{1 / 2}-\frac{\left|\alpha^{(i)}\right|}{\left|\alpha^{(j)}\right|}
$$


Combining (7) and (8) yields

$$
\left|\beta^{(i)}\right| \geq c_{3}(i, j) X-c_{4}(i, j) X^{1 / 2}-c_{5}(i, j),
$$

where

$$
\begin{aligned}
& c_{3}(i, j)=\min \left\{\left|\alpha^{(i)}-\alpha^{(j)}\right|, \frac{\left|\alpha^{(i)}-\alpha^{(j)}\right|}{\left|\alpha^{(j)}\right|}\right\}, \\
& c_{4}(i, j)=\max \left\{2, \frac{\left|\alpha^{(i)}\right|+\left|\alpha^{(j)}\right|}{\left|\alpha^{(j)}\right|}\right\}, \\
& c_{5}(i, j)=\max \left\{1, \frac{\left|\alpha^{(i)}\right|}{\left|\alpha^{(j)}\right|}\right\} .
\end{aligned}
$$

By (6), $X$ is large enough, and thus the expressions on the right side of (9) can be estimated from below by $c_{0}(i, j) X$. Here we may usually put, say, $c_{0}(i, j)=$ $c_{3}(i, j) / 2$, but for our purposes it is useful to make $c_{0}(i, j)$ as large as possible by taking $c_{0}(i, j)=c_{3}(i, j)-\varepsilon$ with, say, $\varepsilon=10^{-2}$ or $10^{-3}$, which can be done in view of (6). Finally, letting $c_{0}=\min _{1 \leq k, l \leq 3, k \neq l} c_{0}(k, l)$, we get

$$
\left|\beta^{(i)}\right| \geq c_{0} X
$$

(In our example, $c_{0}=0.3251256$.)

For simplicity, let $\alpha_{k l}=\alpha^{(k)}-\alpha^{(l)}$ for any $k, l \in\{1,2,3\}$. Let $k=\{1,2,3\} \backslash\{i, j\}$ and consider the following identity:

$$
\alpha_{i j}\left(\beta^{(k)}-\lambda^{(k)}\right)+\alpha_{j k}\left(\beta^{(i)}-\lambda^{(i)}\right)+\alpha_{k i}\left(\beta^{(j)}-\lambda^{(j)}\right)=0 .
$$

Let $\Lambda=\alpha_{i j} \lambda^{(k)}+\alpha_{j k} \lambda^{(i)}+\alpha_{k i} \lambda^{(j)}$; then (11) implies

$$
\frac{\alpha_{i j} \beta^{(k)}}{\alpha_{k j} \beta^{(i)}}=1-\frac{\alpha_{k i} \beta^{(j)}-\Lambda}{\alpha_{k j} \beta^{(i)}}=1-z .
$$

We recall that $\beta=\eta_{1}^{b_{1}} \eta_{2}^{b_{2}}$. From the above equation we obtain

$$
L=\left|b_{1} \log \right| \frac{\eta_{1}^{(k)}}{\eta_{1}^{(i)}}\left|+b_{2} \log \right| \frac{\eta_{2}^{(k)}}{\eta_{2}^{(i)}}|+\log | \frac{\alpha_{i j}}{\alpha_{k j}}||=|\log | 1-z|| .
$$

From (10) we get

$$
|z| \leq \frac{\left|\alpha_{k i}\right|+\left(\left|\alpha_{12}\right|+\left|\alpha_{23}\right|+\left|\alpha_{31}\right|\right) X^{1 / 2}}{\left|\alpha_{k j}\right| c_{0} X} \leq c_{6} X^{-1 / 2}
$$

with $c_{6}=(1+\varepsilon) \cdot\left(\sum_{1 \leq l<m \leq 3}\left|\alpha_{l m}\right|\right) /\left(c_{0} \min _{1 \leq l<m \leq 3}\left|\alpha_{l m}\right|\right)$, where we may put, for example, $\varepsilon=10^{-2}$ or $2 \cdot 10^{-2}$ if (6) ensures $\max _{1 \leq l<m \leq 3}\left|\alpha_{l m}\right|<\varepsilon \cdot X_{0}^{1 / 2}$. In our example, $c_{6}=87.206869$. Using (6) again, we can see that $|z| \leq 1 / 4$ and thus

$$
L=|\log | 1-z||=\left|z+\frac{z^{2}}{2}+\frac{z^{3}}{3}+\cdots\right| \leq \frac{|z|}{|1-z|} \leq \frac{4}{3} c_{6} X^{-1 / 2} .
$$

Since we need an upper bound for $L$ in terms of $H$, we apply (5) to (12) to get

$$
L \leq \exp \left(-c_{7} H+c_{8}\right),
$$

where $c_{7}=c_{1} / 2, c_{8}=\log \left(4 c_{6} / 3\right)+c_{2} / 2 ;$ in our example, $c_{7}=0.1878707, c_{8}=$ 5.2511113 .

To give a lower bound for $L$, we use Baker's method, more precisely, the following sharp estimate of Waldschmidt [24] for linear forms in the logarithms of algebraic numbers. We formulate this result in the special case when $b_{i} \in Q$ (cf. Theorem 3 of $[22])$. 
LEMMA 1 (Waldschmidt [24]). Let $\alpha_{1}, \ldots, \alpha_{n}$ be nonzero algebraic numbers and let $b_{0}, \ldots, b_{n}$ be rational integers. Let $D=\left[Q\left(\alpha_{1}, \ldots, \alpha_{n}\right): Q\right]$, and suppose that $\alpha_{i}$ has defining equation $a_{0} x^{d}+\cdots+a_{d}=0$ where $\left(a_{0}, \ldots, a_{d}\right)=1$. Define the measure of $\alpha_{i}$ by

$$
M\left(\alpha_{i}\right)=a_{0} \prod_{\sigma} \max \left(1,\left|\sigma \alpha_{i}\right|\right),
$$

where $\sigma$ runs through all embeddings of $Q\left(\alpha_{i}\right) \rightarrow C$, and let the absolute logarithmic height of $\alpha_{i}$ be defined by

$$
h\left(\alpha_{i}\right)=\log \left(M\left(\alpha_{i}\right)\right) / D .
$$

Further, let $V_{0}=1 / D$ and $V_{j} \geq \max \left(h\left(\alpha_{j}\right),\left|\log \alpha_{j}\right| / D, V_{j-1}\right)$ for $1 \leq j \leq n$. Finally, let $E$ be any number satisfying

$$
1 \leq E \leq \min \left\{e^{D V_{1}}, \min _{1 \leq j \leq n} \frac{4 D V_{j}}{\left|\log \alpha_{j}\right|}\right\}
$$

and let $V_{j}^{+}=\max \left(V_{j}, 1\right)$ for $j=n$ and $n-1$, with $V_{0}^{+}=1$ if $n=1$. If $\Lambda=$ $b_{0}+b_{1} \log \alpha_{1}+\cdots+b_{n} \log \alpha_{n}$ does not vanish, then

$$
|\Lambda|>\exp [-W(\log H+C)]
$$

where

$$
\begin{gathered}
W=C(n) D^{n+2} V_{1} \cdots V_{n}\left(\log E D V_{n-1}^{+}\right)(\log E)^{-n-1}, \\
H=\max _{0 \leq j \leq n}\left|b_{j}\right|, \quad C=\log \left(E D V_{n}^{+}\right)
\end{gathered}
$$

and

$$
C(1) \leq 2^{35}, \quad C(2) \leq 2^{53}, \quad C(3) \leq 2^{71}, \quad C(n) \leq 2^{8 n+51} n^{2 n} .
$$

We shall apply this deep result for $n=3$ and for the algebraic numbers $\delta_{1}=$ $\left|\eta_{1}^{(k)} / \eta_{1}^{(i)}\right|, \delta_{2}=\left|\eta_{2}^{(k)} / \eta_{2}^{(i)}\right|, \delta_{3}=\left|\alpha_{i j} / \alpha_{k j}\right|$ instead of $\alpha_{1}, \alpha_{2}, \alpha_{3}$. According to the possible choices of $i, j, k$ we have 6 cases, but if we consider $L$, we can easily see that if $j$ is fixed and we interchange the values of $k$ and $i$, then the value of $L$ does not vary. So it is sufficient to consider separately the following cases:

(1.) $j=1, k=2, i=3$,

(2) $j=2, k=1, i=3$,

(3) $j=3, k=1, i=2$.

For the application of Lemma 1 we note that in our case $n=3$ and $D=6$. The leading coefficients of the defining polynomials of $\delta_{1}, \delta_{2}$ are 1 , since they are units in $Q\left(\delta_{1}, \delta_{2}, \delta_{3}\right)$. Further, in our example, the defining polynomial of $\delta_{3}$ is

$$
x^{6}-3 x^{5}-183 x^{4}+371 x^{3}-183 x^{2}-3 x+1
$$

with leading coefficient 1 . Moreover, we calculated

$$
h\left(\delta_{1}\right)=0.8095869, \quad h\left(\delta_{2}\right)=0.46615, \quad h\left(\delta_{3}\right)=0.8831616,
$$

In cases (1.), (2), (3) of our example we obtained the following results:

(1.) $C=3.8992605, \log W=56.004312$,

(2.) $C=5.1708105, \log W=54.39818$,

(3.) $C=3.8712005, \log W=56.050706$. 
We conclude that in any case

$$
L \geq \exp \left(-W_{0}\left(\log H+C_{0}\right)\right)
$$

where $W_{0}$ and $C_{0}$ are the maxima of the values of $W$ and $C$, respectively, obtained in cases (1.), (2.), (3). In our example, $W_{0}=\exp (56.050706), C_{0}=5.1708105$.

Combining (13) and (14), we infer

$$
-W_{0}\left(\log H+C_{0}\right) \leq-c_{7} H+c_{8},
$$

whence

$$
H \leq \frac{W_{0} C_{0}}{c_{7}}+\frac{W_{0}}{c_{7}} \log H+\frac{c_{8}}{c_{7}} .
$$

Let $c_{9}=W_{0} / c_{7}+c_{8} / c_{7}$ and $c_{10}=\max \left\{W_{0} C_{0} / c_{7}, c_{9}\right\}$. Then we obtain

$$
\log \left(\frac{H}{\log H+1}\right) \leq \log c_{10} \text {. }
$$

In our example, $\log c_{10}=59.365737$. We checked inequality (15) for some powers of 10 and we found that it holds for $H=10^{27}$ but fails for $H=10^{28}$, which implies $H \leq 10^{28}$ in our example.

3. Reduction with the Method of Baker and Davenport. In this section we shall reduce the large upper bound obtained for $H$ by Baker's method.

Dividing $L$ by $\log \left|\eta_{2}^{(k)} / \eta_{2}^{(i)}\right|$ and applying (13) we have

$$
L^{\prime}=\left|b_{1} \vartheta+b_{2}-\beta\right| \leq A K^{-H},
$$

where

$$
\begin{gathered}
\vartheta=\frac{\log \left|\eta_{1}^{(k)}\right| /\left|\eta_{1}^{(i)}\right|}{\log \left|\eta_{2}^{(k)}\right| /\left|\eta_{2}^{(i)}\right|}, \quad \beta=\frac{\log \left|\alpha_{k j} / \alpha_{i j}\right|}{\log \left|\eta_{2}^{(k)}\right| /\left|\eta_{2}^{(i)}\right|}, \\
A=\frac{e^{c_{8}}}{\min _{\substack{1 \leq l, m \leq 3 \\
l \neq m}}|\log | \eta_{2}^{(l)}|/| \eta_{2}^{(m)}||}
\end{gathered}
$$

and $K=e^{c_{7}}$. In our example, $\log A=6.2504055$ and $\log K=0.1878707$.

We shall use the Baker-Davenport lemma in the following modified form:

LEMMA 2. Suppose that $\vartheta, \beta$ are given real numbers, $A$ is a given real constant, $M$ and $B(B>6)$ are rational integers. If there exist rational integers $p, q$ satisfying $1 \leq q \leq M B,|\vartheta q-p|<2 / M B$ and $\|q \beta\| \geq 3 / B$, then there is no solution of the inequality

$$
\left|b_{1} \vartheta+b_{2}-\beta\right| \leq A K^{-H}
$$

in rational integers $b_{1}, b_{2}$ with

$$
\frac{\log \left(M B^{2} A\right)}{\log K} \leq H \leq M,
$$

where $H=\max \left(\left|b_{1}\right|,\left|b_{2}\right|\right)$ and $\|\cdot\|$ denotes the distance from the nearest integer.

Proof. The proof involves standard arguments (cf., e.g., [5]).

Put $w=\vartheta-p / q$, so that $|w| \leq 2 / q M B$. We have

$$
\left|b_{1} q \vartheta+b_{2} q-\beta q\right|<q A K^{-H} \leq M B A K^{-H} .
$$


Since $\vartheta q=q w+p$, the above inequality implies

$$
\left|b_{1} p+b_{1} q w+b_{2} q-\beta q\right|<M B A K^{-H}
$$

By the assumptions

$$
\|q \beta\| \geq 3 / B \text { and }\left|b_{1} q w\right| \leq M q \frac{2}{q M B}=2 / B
$$

there follows

$$
\left\|b_{1} q w-q \beta\right\| \geq 1 / B
$$

Thus,

$$
1 / B \leq\left|b_{1} q w-q \beta+b_{1} p+b_{2} q\right|<M B A K^{-H}
$$

which implies

$$
H<\frac{\log \left(M B^{2} A\right)}{\log K} .
$$

This modification of the original lemma is necessary to avoid further diminishing the value of $\log K$ in (16), which would make the reduction procedure less efficient.

We apply Lemma 2 to inequality (16) to reduce the bound $H \leq H_{0}$ (in our example $H_{0}=10^{28}$ ) separately in cases (1.), (2.), (3.).

The reduction, in the case of our example, was done in four steps as shown in the following table.

\begin{tabular}{l|c|r|r|l|c|r}
\hline & $M$ & $B$ & $q \leq$ & $\|\vartheta q\|<$ & $\|\beta q\| \geq$ & new $M$ \\
I & $10^{28}$ & 100 & $10^{30}$ & $2 \cdot 10^{-30}$ & 0.03 & 426 \\
II & 426 & 100 & 42600 & $4.69 \cdot 10^{-5}$ & 0.03 & 115 \\
III & 115 & 50 & 5750 & $3.47 \cdot 10^{-4}$ & 0.06 & 101 \\
IV & 101 & 11 & 1111 & $1.80 \cdot 10^{-3}$ & 0.2727273 & 84 \\
\hline
\end{tabular}

The final purpose was to reduce the bound $H \leq 10^{28}$ to $H \leq 84$.

For this reason we used multiprecision arithmetic to compute the values of $\alpha^{(1)}$, $\alpha^{(2)}, \alpha^{(3)}$ with the accuracy of 100 digits, which enabled us in cases (1), (2), (3) to compute the corresponding values of $\vartheta$ and $\beta$. The approximate values $\vartheta_{0}$ and $\beta_{0}$ obtained from $\alpha^{(1)}, \alpha^{(2)}, \alpha^{(3)}$ by using 100-digit numbers were exact up to at least 90 digits: $\left|\vartheta-\vartheta_{0}\right|<10^{-90},\left|\beta-\beta_{0}\right|<10^{-90}$. Since in cases I-IV, $q \leq 10^{30}$, we have

$$
\|q \vartheta\|=|q \vartheta-p| \leq q\left|\vartheta-\vartheta_{0}\right|+\left|q \vartheta_{0}-p\right| \leq 10^{-60}+\left\|q \vartheta_{0}\right\|,
$$

and similarly $\left\|q \beta_{0}\right\| \leq 10^{-60}+\|q \beta\|$, that is, $\|q \beta\| \geq\left\|q \beta_{0}\right\|-10^{-60}$. This shows that using the values of $\vartheta_{0}, \beta_{0}$ we have to calculate integers $q$ so that $\left\|q \vartheta_{0}\right\|,\left\|q \beta_{0}\right\|$ must satisfy somewhat sharper inequalities than the corresponding inequalities for $\|q \vartheta\|,\|q \beta\|$ in the above table. That is, we have to find $q$ such that

$$
\left\|q \vartheta_{0}\right\|<\frac{2}{M B}-10^{-60}, \quad\left\|q \beta_{0}\right\| \geq \frac{3}{B}+10^{-60}
$$


As usual, we computed the values of $q$ using the continued fraction expansion of $\vartheta_{0}$, in which case the corresponding inequalities were satisfied with much greater margin, not merely with a margin of $10^{-60}$. In cases (1.), (2.), (3.) we obtained the following values for $q$ :

I.

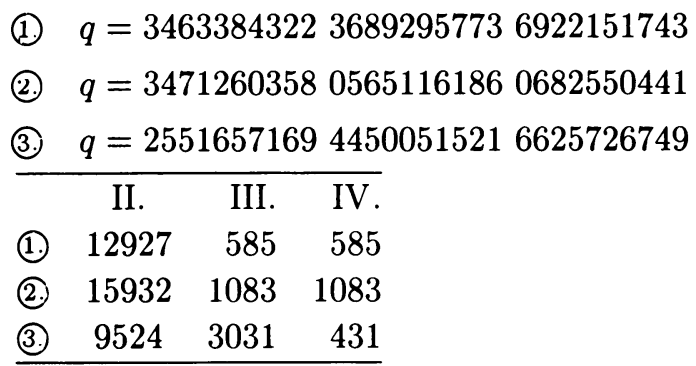

In this way we obtained a new and rather low bound $H_{M}$ for $H$ : in our example, $H_{M}=84$.

4. Solutions of Medium Size. We now turn to the range $H_{0}<H \leq H_{M}$ (in our example, $H_{0}=35, H_{M}=84$ ). In the following we show how one can reduce the procedure of checking the pairs $b_{1}, b_{2}$ in this range to the test of very few cases only.

Let $H_{1}<H_{2}$ be integers in the interval $\left(H_{0}, H_{M}\right]$ and consider the pairs of exponents $b_{1}, b_{2}$ with $H_{1} \leq H \leq H_{2}$, where $H=\max \left(\left|b_{1}\right|,\left|b_{2}\right|\right)$ as before. $b_{1}, b_{2}$ must satisfy (16), that is

$$
\left|b_{1} \vartheta+b_{2}-\beta\right|<A K^{-H} \leq A K^{-H_{1}},
$$

whence

$$
-A K^{-H_{1}}-b_{1} \vartheta+\beta \leq b_{2} \leq A K^{-H_{1}}-b_{1} \vartheta+\beta
$$

We remark that here we have three possibilities for the values of $\vartheta$ and $\beta$ corresponding to the cases (1.), (2.), (3.). If $H_{1}$ is sufficiently large, then $A K^{-H_{1}}$ has a small value, which means that there may be only very few possible values of $b_{2}$ for a fixed $b_{1}$.

To get all possible pairs $b_{1}, b_{2}$ with $H_{1} \leq H \leq H_{2}$, we let $b_{1}$ run through all integers in $\left[-H_{2}, H_{2}\right]$. For each $b_{1}$ we determine the possible values of $b_{2}$ from (17) according to the cases (1.), (2.), (3.). We discard the pair $b_{1}, b_{2}$ if $H_{1} \leq H \leq H_{2}$ does not hold or if $\left|b_{1} \vartheta+b_{2}-\beta\right| \geq A K^{-H}$.

In our example we used the following values in the role of $H_{1}, H_{2}$ :

\begin{tabular}{l|l|l|l|l|l}
\hline$H_{1}$ & 36 & 40 & 45 & 55 & 65 \\
$H_{2}$ & 39 & 44 & 54 & 64 & 85 \\
\hline
\end{tabular}

The intervals $\left[H_{1}, H_{2}\right]$ covered the range $\left(H_{0}, H_{M}\right]$ completely and we found only 24 possible pairs $b_{1}, b_{2}$, which were tested with the algorithms described in Section 5 . 
5. Searching Over the Remaining Set. In this section we describe those algorithms which enabled us to check the possible pairs $b_{1}, b_{2}$. These algorithms were used to test all pairs in the range $H \leq H_{0}$ and the remaining few pairs with $H_{0}<H \leq H_{M}$ found in Section 4 .

Consider a fixed pair $b_{1}, b_{2}$. We have to decide whether $\beta=\eta_{1}^{b_{1}} \eta_{2}^{b_{2}}$ may have the form $x+\alpha y+\lambda$ with $x, y \in Z, \lambda \in Z_{K}$, where $|\lambda|<X^{1 / 2}, X=\max (|x|,|y|)$. Our condition on $\lambda$ can be written in the form

$$
\max _{1 \leq i \leq 3}\left|\beta^{(i)}-x-\alpha^{(i)} y\right| \leq \max (\sqrt{|x|}, \sqrt{|y|})
$$

where $x, y \in Z$ are unknowns, but $\beta^{(i)}$ is given along with $b_{1}, b_{2}$.

I. If $|x| \geq|y|$ then (18) yields

$$
-\sqrt{|x|} \leq \beta^{(i)}-x-\alpha^{(i)} y \leq \sqrt{|x|}
$$

whence

$$
-\frac{x}{\alpha^{(i)}}-\frac{\sqrt{|x|}}{\left|\alpha^{(i)}\right|}+\frac{\beta^{(i)}}{\alpha^{(i)}} \leq y \leq-\frac{x}{\alpha^{(i)}}+\frac{\sqrt{|x|}}{\left|\alpha^{(i)}\right|}+\frac{\beta^{(i)}}{\alpha^{(i)}} \quad(i=1,2,3) .
$$

II. If $|x|<|y|$ then (18) implies

$$
-\sqrt{|y|} \leq \beta^{(i)}-x-\alpha^{(i)} y \leq \sqrt{|y|}
$$

that is,

$$
-\alpha^{(i)} y-\sqrt{|y|}+\beta^{(i)} \leq x \leq-\alpha^{(i)} y+\sqrt{|y|}+\beta^{(i)} \quad(i=1,2,3) .
$$

If in (20) we interchange the roles of $x$ and $y$, we can see that in both cases I, II we want to find rational integers $x$ and $y$ satisfying the system of inequalities

$$
A_{i} x-B_{i} \sqrt{|x|}+C_{i} \leq y \leq A_{i} x+B_{i} \sqrt{|x|}+C_{i} \quad(i=1,2,3),
$$

where the coefficients $A_{i}, B_{i}, C_{i}$ are given by (19) and (20). (In both cases, $B_{i}>0$, $i=1,2,3$.) Further (after the change of $x$ and $y$ in case II), $x$ and $y$ must satisfy

$$
-|x| \leq y \leq|x|
$$

as well.

First, we have to find those values of $x$ for which the intersection of the intervals given by (21) for $y$ is nonempty. This is the case only if any lower bound, given for $y$ in terms of $x$, is less than any such upper bound, that is, if

$$
\left(A_{i}-A_{j}\right) x-\left(B_{i}+B_{j}\right) \sqrt{|x|}+\left(C_{i}-C_{j}\right) \leq 0 \quad(1 \leq i, j \leq 3, i \neq j)
$$

is satisfied. (In view of $B_{i}>0$, the similar inequality for $i=j$ is trivial.) These inequalities are second-degree inequalities for $\sqrt{|x|}$.

The main problem of this section is to find the solutions of (23). Here, the coefficients $A_{i}, B_{i}$ have (in most cases and also in our example) moderate values. The difficulty is caused by the values of $C_{i}$, which may be very high or low compared 
to $A_{i}$ and $B_{i}$. Of course, the solutions of the system of inequalities (23) could be found in a unique manner by using multiple-precision arithmetic, but since it is necessary to use a procedure for computing square roots, which is an iteration procedure requiring a considerable amount of computation time, it is better to avoid multiple-precision arithmetic if at all possible. Since large values of $C_{i}$ cannot be exactly represented in single precision, we used, given the magnitude of the $C_{i}$, two algorithms to test all pairs of exponents $b_{1}, b_{2}$.

Our first method was applicable when all the $C_{i}$ were small, say $\left|C_{i}\right|<10^{6}$. In this case (in which we expect the solutions anyhow) there is no difficulty in determining the solutions of the system of inequalities (23). We examined cases I and II separately and in both of them two subcases according as $x \geq 0$ or $x<0$. In each subcase we proceeded as follows:

a. For each inequality in (23) we computed the intervals in which $\sqrt{|x|}$ may assume its values and we took the intersection of this set of intervals.

b. We transformed the intervals (obtained by taking intersection) for $\sqrt{|x|}$ into intervals for $x$, and for all integers $x$ in this set of intervals we calculated the maximum $M A$ of the lower bounds and the minimum $M I$ of the upper bounds given by (21) for $y$ in terms of $x$. For fixed $x$, the corresponding values of $y$ are the integers lying in $[M A, M I] \cap[-|x|,|x|]$ (cf. (22)).

In this procedure, if in any step the calculated interval (system) is empty, we pass to the next subcase.

Now consider those cases in which some of the $\left|C_{i}\right|$ are large $\left(>10^{6}\right)$. In this case (when we do not expect any solutions) our purpose is to eliminate the cases by a simple method.

Let $A=\max \left|A_{i}-A_{j}\right|, a=\min \left|A_{i}-A_{j}\right|, B=\max \left|B_{i}+B_{j}\right|, A_{0}=\max \left|A_{i}\right|$, $B_{0}=\max \left|B_{i}\right|$, where the maxima and minima are taken for cases I, II and for each $i \neq j$ in $A, a, B$ and for each $i$ in $A_{0}, B_{0}$. The following lemma allowed us to eliminate easily a considerable amount of pairs of exponents $b_{1}, b_{2}$.

LEMMA 3. Suppose that for fixed $b_{1}, b_{2}$ in case I (resp. in case II) there exist indices $i, j, k(i \neq j)$ and positive constants $D_{0}$ and $K$ with the following properties:

$$
\left|C_{k}\right|>D_{0}, \quad\left|C_{i}-C_{j}\right|<K \quad \text { and } \quad D_{1}^{2}(K)+D_{2}(K)<D_{0},
$$

where $D_{1}(K)=\left(B+\sqrt{B^{2}+4 A K}\right) / 2 a$ and $D_{2}(K)=A_{0} D_{1}^{2}(K)+B_{0} D_{1}(K)$. Then there are no solutions in case I (resp. case II).

Proof. We may assume that $A_{i}-A_{j}>0$ since otherwise the inequality will hold after an interchange of $i$ and $j$. Then, if $x>0,(23)$ is a second-degree inequality for $\sqrt{|x|}$, whence $\sqrt{|x|} \leq D_{1}(K)$. (If $x<0$, the same holds after an interchange of $i$ and $j$.) Thus,

$$
\left|A_{k} x \pm B_{k} \sqrt{|x|}\right| \leq D_{2}(K) .
$$

In view of (21) and (22), the possible values of $y$ must satisfy

$$
|y| \geq\left|A_{k} x \mp B_{k} \sqrt{|x|}+C_{k}\right| \geq D_{0}-D_{2}(K)
$$


and

$$
|y| \leq|x| \leq D_{1}^{2}(K)
$$

(In the above inequality we take the sign - or + according as $C_{k}>0$ or $C_{k}<0$.) Now if $D_{1}^{2}(K)<D_{0}-D_{2}(K)$, then the contradiction shows that there cannot be any solutions.

In our example, we applied Lemma 3 with $D_{0}=10^{6}, K=4520$. In our case we have $\left|\eta_{1}^{(2)}\right|>1,\left|\eta_{2}^{(2)}\right|>1$, while $\left|\eta_{1}^{(1)}\right|,\left|\eta_{1}^{(3)}\right|,\left|\eta_{2}^{(1)}\right|,\left|\eta_{2}^{(3)}\right|$ are less than 1 . (One can always choose fundamental units with this property.) Considering the numerical values of the conjugates of our units, we can see that, if $b_{1} \geq 11$ and $b_{2} \geq 0$, then the conditions of Lemma 3 are satisfied (with $k=2, i=1, j=3$ ), so these pairs could be discarded without any test.

In our second algorithm we dealt with those cases in which large values of the $C_{i}$ occurred. In these cases, $\operatorname{sgn}\left(C_{i}\right)$ and $\log \left|C_{i}\right|$ was represented in the computer instead of $C_{i}$. Addition of such values was performed by a separate subroutine which computed upper and lower estimates of the sum. In each case, when Lemma 3 was not applicable, we followed step a. of our first algorithm, but using logarithmic representation of numbers and being careful to round up or down the results of additions in order to get somewhat wider intervals. If the intersection of the set of intervals was not empty, then we obtained a maximal value $X M$ for $\sqrt{|x|}$. Afterwards, we calculated the upper estimate $X U=A_{0} X M^{2}+B_{0} X M$ for $\left|A_{i} x \pm B_{i} \sqrt{|x|}\right|$ and each case could be eliminated by showing that the intersection of the intervals $\left[C_{i}-X U, C_{i}+X U\right](i=1,2,3)$ and $\left[-X M^{2}, X M^{2}\right]$ is empty.

6. Computational Aspects. The first version of the program was developed on a simple C64, but later the same programs were executed on an IBM PC compatible computer for which the computation time took only minutes. The multipleprecision arithmetic for the reduction procedure was written in Pascal, using the classical algorithms of Knuth [14]. The testing algorithms described in Section 5 were written in Basic. The test of the remaining set (appr. 4000 pairs $b_{1}, b_{2}$ ) took about half of the computation time.

7. List of Solutions. Finally, we give all solutions of the equation which was our example throughout the paper. In each case we give the pair of exponents $b_{1}, b_{2}$, the coordinates of $\beta=\eta_{1}^{b_{1}} \eta_{2}^{b_{2}}$ in the integral base $1, \alpha, \alpha^{2}$ of $Z_{K}$ and the values of $x$ and $y$. These data make possible to calculate the coordinates of $\lambda$ corresponding to $x$ and $y$. Naturally, in the table one can find also all solutions of the corresponding homogeneous equation

$$
N_{K / Q}(x+\alpha y)=x^{3}+3 x^{2} y-4 x y^{2}+y^{3}=1 .
$$

A Thue equation equivalent to this one was already solved by Pethö and Schulenberg [19]. In the inhomogeneous case, there are usually further solutions corresponding to a solution of the homogeneous equation, but there are also several other solutions 
which do not correspond to any solution of the homogeneous equation.

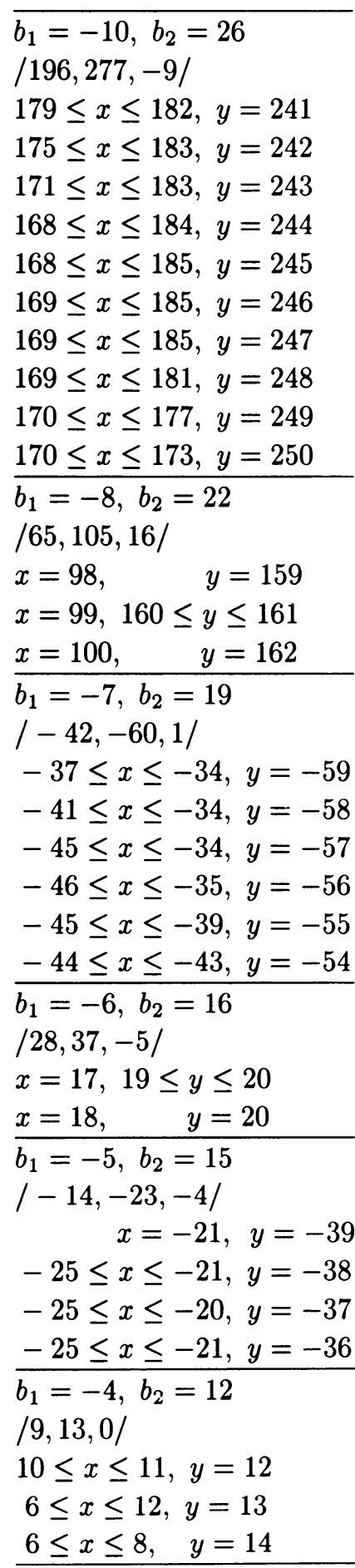

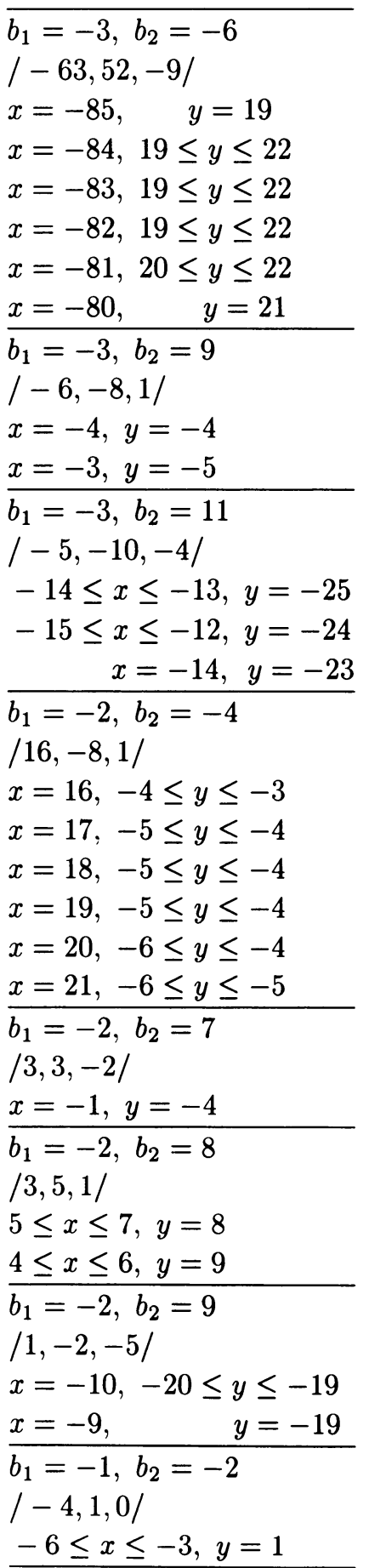




\begin{tabular}{|c|c|}
\hline$b_{1}=-1, b_{2}=5$ & \\
\hline $\begin{array}{l}-2,-3,0 \\
-3 \leq x \leq-1, y=-3\end{array}$ & $b_{1}=1, b_{2}=3$ \\
\hline $\begin{array}{l}b_{1}=-1, b_{2}=6 \\
/-1,0,2 /\end{array}$ & $\begin{array}{l}/-1,-3,-2 / \\
-6 \leq x \leq-4, y=-10\end{array}$ \\
\hline $3 \leq x \leq 4, y=7$ & $b_{1}=1, b_{2}=4$ \\
\hline $\begin{array}{l}b_{1}=-1, b_{2}=7 \\
/-2,-5,-3 /\end{array}$ & $\begin{array}{l}/ 1,5,5 / \\
x=11, \quad y=22 \\
x=12,22 \leq y \leq 23\end{array}$ \\
\hline $\begin{array}{r}-10 \leq x \leq-8, y=-16 \\
-9 \leq x \leq-7, y=-15 \\
\end{array}$ & $b_{1}=2, b_{2}=0$ \\
\hline$b_{1}=0, b_{2}=0$ & $\begin{array}{l}/ 0,0,1 / \\
x=2, y=4\end{array}$ \\
\hline $1 \leq x \leq 2, y=0$ & $b_{1}=2, b_{2}=1$ \\
\hline$b_{1}=0, b_{2}=2$ & $\begin{array}{l}/ 0,-1,-2 / \\
-5 \leq x \leq-3, y=-8\end{array}$ \\
\hline & $b_{1}=2, b_{2}=2$ \\
\hline$b_{1}=0, b_{2}=4$ & $\begin{array}{r}/ 1,4,4 / \\
x=10, y=17\end{array}$ \\
\hline $\begin{array}{l}11,2,1 / \\
\quad x=4, y=5\end{array}$ & $\begin{array}{l}9 \leq x \leq 10, y=18 \\
9 \leq x \leq 10, y=19\end{array}$ \\
\hline $2 \leq x \leq 3, \quad y=6$ & $b_{1}=3, b_{2}=-2$ \\
\hline $\begin{array}{l}b_{1}=0, b_{2}=5 \\
/ 0,-2,-3 /\end{array}$ & $\begin{array}{l}/ 1,3,0 / \\
0 \leq x \leq 2, y=3\end{array}$ \\
\hline$-7 \leq x \leq-6, y=-12$ & $b_{1}=3, b_{2}=-1$ \\
\hline $\begin{array}{l}b_{1}=0, b_{2}=6 \\
/ 2,7,6 / \\
x=15,27 \leq y \leq 29\end{array}$ & $\begin{array}{l}-1,-3,-1 / \\
-3 \leq x \leq-2, y=-7 \\
-4 \leq x \leq-3, y=-6 \\
\end{array}$ \\
\hline$x=16, \quad y=29$ & $b_{1}=3, b_{2}=0$ \\
\hline $\begin{array}{l}b_{1}=1, b_{2}=0 \\
/ 0,1,0 / \\
x=0, y=1\end{array}$ & $\begin{array}{l}6 \leq x \leq 8, y=14 \\
7 \leq x \leq 9, y=15\end{array}$ \\
\hline $\begin{array}{l}b_{1}=1, b_{2}=1 \\
/-1,-2,0 / \\
-2 \leq x \leq 0, y=-2\end{array}$ & $\begin{array}{l}b_{1}=3, b_{2}=1 \\
/-2,-8,-7 / \\
x=-17,-33 \leq y \leq-32 \\
\end{array}$ \\
\hline $\begin{array}{l}b_{1}=1, b_{2}=2 \\
/ 0,1,1 / \\
x=3, y=4\end{array}$ & $\begin{array}{l}b_{1}=4, b_{2}=-3 \\
/ 1,2,-2 / \\
x=-3, y=-5\end{array}$ \\
\hline
\end{tabular}

$x=2, y=5$ 


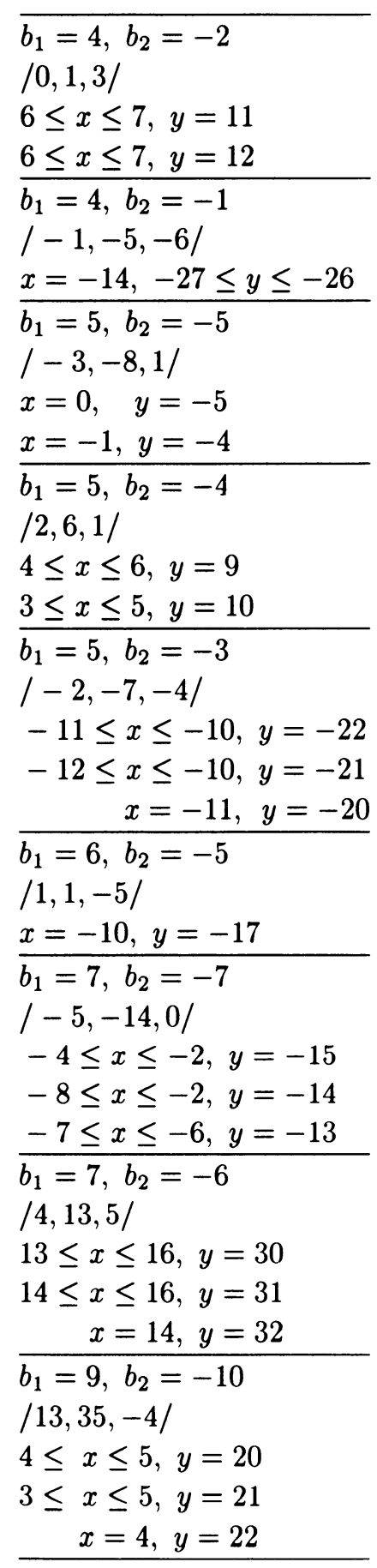

$b_{1}=9, b_{2}=-9$

/ $-9,-27,-5 /$

$-20 \leq x \leq-18, y=-46$

$-22 \leq x \leq-18, y=-45$

$-22 \leq x \leq-17, y=-44$

$-21 \leq x \leq-20, y=-43$

$b_{1}=11, b_{2}=-12$

$/ 22,62,1 /$

$27 \leq x \leq 30, y=63$

$23 \leq x \leq 30, y=64$

$19 \leq x \leq 31, y=65$

$18 \leq x \leq 30, y=66$

$18 \leq x \leq 26, y=67$

$19 \leq x \leq 22, y=68$

$b_{1}=15, b_{2}=-17$

/ $-97,-275,-9$ /

$-112 \leq x \leq-110, y=-312$

$-116 \leq x \leq-109, y=-311$

$-120 \leq x \leq-108, y=-310$

$-124 \leq x \leq-108, y=-309$

$-127 \leq x \leq-107, y=-308$

$-127 \leq x \leq-106, y=-307$

$-126 \leq x \leq-106, y=-306$

$-126 \leq x \leq-106, y=-305$

$-125 \leq x \leq-110, y=-304$

$-125 \leq x \leq-114, y=-303$

$-125 \leq x \leq-118, y=-302$

$-124 \leq x \leq-122, y=-301$

Acknowledgments. I am thankful to Attila Pethö for his help in the preparation of this paper. The idea used in Section 4 to reduce the number of pairs $b_{1}, b_{2}$ to be tested in the range $H_{0}<H \leq H_{M}$ is due to him. 
Kossuth Lajos University

Mathematical Institute

Debrecen, Pf. 12

H-4010, Hungary

1. A. BAKer, "Contributions to the theory of Diophantine equations," Philos. Trans. Roy. Soc. London Ser. A, v. 263, 1968, pp. 173-208.

2. A. BAKER, Transcendental Number Theory, 2nd ed., Cambridge Univ. Press, New York, 1979.

3. A. BAKER \& H. DAVENPORT, "The equations $3 x^{2}-2=y^{2}$ and $8 x^{2}-7=z^{2}$," Quart. $J$. Math. Oxford, v. 20, 1969, pp. 129-137.

4. J. CoAtes, "An effective $p$-adic analogue of a theorem of Thue," Acta Arith., v. 15, 1969, pp. 279-305.

5. W. J. ElLiSON, "Recipes for solving Diophantine problems by Baker's method," Séminaire de Théorie des Nombres, 1970-1971, Exp. No. 11, Lab. Théorie des Nombres, C.N.R.S., Talence, 1971.

6. W. J. Ellison, F. Ellison, J. Pesek, C. E. Stahl \& D. S. Stall, "The Diophantine equation $y^{2}+k=x^{3}$," J. Number Theory, v. 4, 1972, pp. 107-117.

7. I. GAÁL, "Norm form equations with several dominating variables and explicit lower bounds for inhomogeneous linear forms with algebraic coefficients," Studia Sci. Math. Hungar., v. 19, 1984, pp. 399-411.

8. I. GAÁL, "Norm form equations with several dominating variables and explicit lower bounds for inhomogeneous linear forms with algebraic coefficients II," Studia Sci. Math. Hungar., v. 20, 1985, pp. 333-344.

9. K. GYÖRY, "Sur certaines généralisations de l'équation de Thue-Mahler," Enseign. Math., v. 26,1980 , pp. $247-255$.

10. K. GYÖRY, Résultats Effectifs sur la Représentation des Entiers par des Formes Décomposables, Queen's Papers in Pure and Appl. Math., No. 56, Kingston, Ontario, Canada, 1980.

11. K. GYÖRY, "On $S$-integral solutions of norm form, discriminant form and index form equations," Studia Sci. Math. Hungar., v. 16, 1981, pp. 149-161.

12. K. GYÖRY, "Bounds for the solutions of norm form, discriminant form and index form equations in finitely generated integral domains," Acta Math. Hungar., v. 42, 1983, pp. 45-80.

13. K. GYÖRY \& Z. Z. PAPP, Norm Form Equations and Explicit Lower Bounds for Linear Forms with Algebraic Coefficients, Studies in Pure Math. (To the memory of Paul Turán), Akadémiai Kiado, Budapest, 1983, pp. 245-257.

14. D. E. KNUth, The Art of Computer Programming, II, Addison-Wesley, Reading, Mass., 1966.

15. S. V. Kotov, On Diophantine Equations of Norm Form Type II, Inst. Mat. Akad. Nauk BSSR, Preprint No. 10, Minsk, 1980. (Russian)

16. S. V. Kotov, Effective Bounds for Linear Forms with Algebraic Coefficients in Archimedean and p-Adic Metrics, Inst. Mat. Akad. Nauk BSSR, Preprint No. 24, Minsk, 1981. (Russian)

17. S. V. Kotov, "Effective bound for the values of the solutions of a class of Diophantine equations of norm form type," Mat. Zametki, v. 33, 1983, pp. 801-806. (Russian)

18. A. Ретнӧ, "On the resolution of Thue inequalities," J. Symb. Comput., v. 4, 1987, pp. 103-109.

19. A. Pethö \& R. Schulenberg, Effektives Lösen von Thue Gleichungen, Publ. Math. Debrecen, v. 34, 1987, pp. 189-196.

20. T. N. ShOREY \& R. TIJDEMAn, Exponential Diophantine Equations, Cambridge Univ. Press, Oxford, 1986.

21. V. G. SPRINDŽUK, "Representation of numbers by the norm forms with two dominating variables," J. Number Theory, v. 6, 1974, pp. 481-486.

22. R. P. StEINER, "On Mordell's equation $y^{2}-k=x^{3}$ : A problem of Stolarsky," Math. Comp., v. 46,1986, pp. $703-714$.

23. R. TijDeman, On the Gel'fond-Baker Method and Its Application, Proc. Sympos. Pure Math., vol. 28, Amer. Math. Soc., Providence, R.I., 1976, pp. 241-268.

24. M. WALDSChMiDT, "A lower bound for linear forms in logarithms," Acta Arith., v. 37, 1980, pp. $257-283$. 